\title{
BIOPOLÍTICA E REJUVENESCIMENTO: UMA REVISÃO SISTEMÁTICA SOBRE OS DISCURSOS CIENTÍFICOS NA EDUCAÇÃO FÍSICA
}

\author{
Fábio Luís Santos Teixeira \\ Universidade Federal da Paraíba, Paraíba, João Pessoa, Brasil \\ Dênis Oliveira Decussatti \\ Faculdade Maurício de Nassau, Paraíba, João Pessoa, Brasil \\ Rita de Cássia Paulino Silva \\ Universidade Federal da Paraíba, Paraíba, João Pessoa, Brasil \\ Iraquitan Oliveira Caminha \\ Universidade Federal da Paraíba, Paraíba, João Pessoa, Brasil
}

\begin{abstract}
Resumo
Constata-se recentemente que a Educação Física tem se articulado com os discursos biopolíticos de rejuvenescimento desempenhando efeitos de poder na medida em que ela nos oferece a possibilidade de construir um corpo resistente ao envelhecimento. Os discursos científicos da Educação Física parecem corroborar para estabelecimento de vínculos entre saberes e práticas de poder sobre o rejuvenescimento. Dessa maneira, a seguinte revisão sistemática tem como objetivos analisar o processo de constituição da Educação Física como discurso e prática de poder sobre o rejuvenescimento, identificando as narrativas sobre o rejuvenescimento corporal nas revistas científicas, e verificando como estas narrativas se articulam aos saberes da Educação Física.
\end{abstract}

Palavras-chave: Biopolítica. Rejuvenescimento. Educação Física.

\section{Introdução}

Ao longo dos últimos séculos, o funcionamento das sociedades ocidentais foi marcado pela instauração de uma razão instrumental como regime de verdade sobre a vida humana (WEBER, 2004; HEIDEGGER, 2007). Na teoria de Foucault (2006), o controle social derivado desse processo ganhou contornos especiais desde o século XVIII, principalmente porque os corpos dos indivíduos e da população passaram a ser encarados como um problema político. Essa estrutura política/ governamental concentrada no domínio do corpo foi denominado de biopolítica.

A biopolítica indica a ação de um poder sobre a vida (biopoder) desenvolvido em nível estatal/institucional para mecanizar gestos, educar ou promover subjetivação ostensiva, garantindo otimização do controle social. Segundo Foucault (2006), a biopolítica promove a criação de saberes, práticas e instituições que compõem o novo cenário de dominação que é caracterizado pela ideia de fazer viver e deixar morrer.

Contudo, atualmente a biopolítica não desempenha efeitos apenas no nível das 
políticas públicas de estado - como se pensava antigamente em relação a problemas como natalidade, mortalidade, incapacidades biológicas e psicológicas e as epidemias nos centros urbanos. É possível verificar, na leitura foucaultiana, que a biopolítica determina relações microfísicas que influenciam os sujeitos a investir sobre eles mesmos de forma a potencializar suas capacidades produtivas. Além disso, a biopolítica produz e divulga saberes determinando a nossa forma de compreender e se relacionar com o mundo.

Todavia, constata-se recentemente que a Educação Física tem se articulado com os discursos de rejuvenescimento desempenhando efeitos de poder na medida em que ela nos oferece a possibilidade de construir um corpo resistente ao envelhecimento (COSTA; VENÂNCIO, 2004; NAKAJIMA et. al, 2011; TAKAYAMA, 2012). Com isso corre-se o risco de que os discursos científicos da Educação Física reforcem nos sujeitos contemporâneos uma valorização do uso de tecnologias sobre o corpo, influenciados por uma cultura do rejuvenescimento, segundo a qual devemos controlar o envelhecimento para prolongar nossa capacidade de produzir.

Ao se apresentar como tecnologia de rejuvenescimento, a Educação Física assegura sua função de mecanismo de controle, passando a contribuir significativamente para uma substituição do discurso sobre "envelhecer bem" pela compreensão de que envelhecimento é algo a ser evitado.

Parece-nos que os discursos científicos da Educação Física atuam intensificando de maneira direta a ideia de rejuvenescimento. $\mathrm{O}$ estudo desses discursos pode esclarecer certos efeitos de subjetivação que precisam ser verificados para pensar os caminhos da própria Educação Física. Por outro lado, podem revelar a ascensão de uma vontade de administrar a vida resistente às exclusões historicamente impostas ao modo de viver o corpo.

Com base no processo histórico de constituição da Educação Física como discurso e prática de poder sobre o rejuvenescimento, considerando aspectos paradoxais da biopolítica como poder, dominação e resistência, o presente estudo objetiva compreender como as narrativas sobre o rejuvenescimento corporal se manifestam nas publicações científicas da área em questão.

Os discursos científicos corroboram para estabelecimento de vínculos entre saberes e práticas de poder sendo que estas práticas expressam significados com efeitos reais sobre o próprio conhecimento e as maneiras de conceber o corpo. Dessa maneira, buscamos especificamente identificar as narrativas sobre o rejuvenescimento corporal nas revistas científicas, e verificar como essas narrativas se articulam aos saberes da Educação Física.

Para isso, realizamos uma revisão sistemática em bibliotecas virtuais de significativa expressão no universo da pesquisa científica (SCIELO, periódicos CAPES, BIREME) no intuito de analisar os artigos produzidos sobre os temas "atividade física", "exercício físico" e "rejuvenescimento" à luz do conceito foucaultiano de biopolítica. O texto a seguir se configura a partir da exposição do método utilizado, passando pela análise e discussão dos dados, a qual se estrutura em três momentos fundamentais. Na sequência, concluímos a reflexão apresentando nossas considerações finais.

\section{Método}

A seguinte pesquisa é de natureza qualitativa e segue a perspectiva da revisão sistemática, tipo de estudo que identifica, seleciona e avalia criticamente estudos considerados relevantes, fornecendo suporte teórico-prático para a classificação e análise de dados 
bibliográficos (LIBERALI, 2011).

Para sua realização, optamos por acessar três bibliotecas virtuais: SCIELO (Scientific Eletrônic Library Online), periódicos da CAPES (portal de periódicos) e BIREME (Biblioteca virtual em saúde). A primeira etapa consistiu em realizar uma exploração na ferramenta DeCS (Descritores em Ciência de Saúde), um vocabulário de termos científicos específicos da área da saúde que se encontra vinculado à BIREME, para definir as palavraschave mais adequadas para realização da pesquisa.

No conjunto dos termos indexados foi possível identificar a presença dos descritores "rejuvenescimento", "rejuvenation", "exercício físico", "physical exercise", "atividade física" e "physical activity", os quais foram utilizados na fase de levantamento de dados. Contudo, após um primeiro levantamento, percebemos a necessidade de expandir a quantidade de descritores. Assim, adotamos o termo "anti-aging", "antienvelhecimento", devido à quantidade de estudos sobre as práticas tecnológicas de rejuvenescimento que empregam este descritor.

Uma vez selecionados os descritores, iniciamos formalmente a fase de coleta de artigos cruzando os descritores nas bibliotecas virtuais. $\mathrm{Na}$ biblioteca virtual BIREME a pesquisa realizada a partir do cruzamento dos descritores "anti-aging" e "physical exercise", e "anti-aging" e "physical activity" resultou num total de 13 e 12 artigos consecutivamente. Após a aplicação de nossos critérios de exclusão, foram descartados, para cada cruzamento acima citado, um total de dez artigos por se tratarem de estudos realizados com animais, por não se enquadraram no recorte de tempo ou por não tratar dos temas como: exercício físico ou atividade física (critérios de exclusão). Houve a exclusão de um artigo por se repetir. No total obtivemos quatro artigos.

Em relação às combinações "rejuvenation" e "physical exercise", e "rejuvenation" e "physical activity" referente à BIREME obtivemos nove ocorrências para primeiro caso e nenhuma ocorrência para o segundo. Os critérios de exclusão permitiram a seleção de apenas sete artigos. Depois de realizar o cruzamento entre os resultados das duas pesquisas realizadas na BIREME, finalmente foram selecionados seis artigos.

A busca realizada na biblioteca virtual SCIELO, resultou em ausência de ocorrências para os descritores selecionados. Acreditamos que isso se deve ao fato de que os descritores foram utilizados em língua inglesa. Dado que a SCIELO reúne pesquisas publicadas em periódicos científicos brasileiros, acreditamos que a ausência de resultados esta relacionado ao idioma.

Na biblioteca virtual Periódicos CAPES a busca ocorreu em duas etapas: 1) combinação dos descritores "anti-aging" e "physical exercise", e "anti-aging" e "physical activity"; 2) combinação dos descritores "rejuvenation" e "physical exercise", e "rejuvenation" e "physical activity". Após a primeira pesquisa, obtivemos 237 artigos para a combinação "anti-aging" e "physical exercise." Para os descritores "anti-aging" e "physical activity" foram obtidos 578 artigos. Com os descritores "rejuvenation" e "physical exercise" foram encontrados 543 artigos e para as palavras "rejuvenation" e "physical activity", foram obtidos 1.338 artigos.

Em seguida, aplicamos nossos critérios de exclusão de forma a remover estudos realizados com animais, estudos fora do recorte de tempo selecionado e estudos que não tratam dos temas exercício físico ou atividade física. Realizamos, ainda, um refinamento por período de publicação, tipo de publicação e idioma. Na última etapa, verificamos se houve repetição de estudos e realizamos uma leitura dos títulos e resumos dos textos. Esse 
procedimento resultou num total final de oito estudos.

Para analisar os dados utilizamos a análise de discurso na perspectiva foucaultiana que enfatiza a identificação e discussão de enunciados que compõem um determinado campo de acontecimentos e que funcionam como funções de existência, ou seja, determinam perspectivas de realidade. No tópico a seguir, realizamos a análise e discussão dos resultados obtidos.

\section{Resultados e discussão}

Os artigos selecionados nas bibliotecas virtuais consultadas foram organizados nas tabelas 1 e 2 para melhor visualização. Em cada tabela destacamos informações gerais sobre as obras analisadas. Isso possibilitou a realização de uma compreensão mais voltada à identificação de diferentes enunciados.

Tabela 1 - Estudos selecionados a partir da pesquisa na biblioteca virtual BIREME.

\begin{tabular}{|c|c|c|c|}
\hline TITTULO & AUTOR & REVISTA & OBJETIVOS \\
\hline $\begin{array}{l}\text { Physical activity and aging: } \\
\text { opposing physiologic effects.* }\end{array}$ & $\begin{array}{c}\text { Charansonney, } \\
\text { O. }\end{array}$ & $\begin{array}{l}\text { Ann. Cardiol. } \\
\text { Angeiol., } 2012 \text {. }\end{array}$ & $\begin{array}{l}\text { Apresentar os efeitos contrários da atividade } \\
\text { física ao envelhecimento a partir de dois } \\
\text { modelos teóricos. }\end{array}$ \\
\hline $\begin{array}{l}\text { Rejuvenescimento facial por } \\
\text { intervenção miofuncional } \\
\text { estética. Revisão integrativa. }\end{array}$ & Souza, C. et al. & $\begin{array}{l}\text { Med. Cutan. Iber. } \\
\text { Lat. A.m., } 2013 \text {. }\end{array}$ & $\begin{array}{l}\text { Analisar }{ }^{\circ} \text { rejuvenescimento facial } \\
\text { decorrente da intervençấo miofuncional } \\
\text { estética. }\end{array}$ \\
\hline $\begin{array}{l}\text { Scientific overview of } \\
\text { hormone treatment used for } \\
\text { rejuvenation. }\end{array}$ & Morley, J. & Fertil Steril, 2013. & $\begin{array}{l}\text { Discutir os efeitos de hormônios em pessoas } \\
\text { idosas com deficiência hormonal. }\end{array}$ \\
\hline $\begin{array}{l}\text { Aerobic physical exercise and } \\
\text { arterial de-stiffening: a recipe } \\
\text { for vascular rejuvenation? }\end{array}$ & Pucci, G. et al. & $\begin{array}{l}\text { Hypertens. Res., } \\
2012 \text {. }\end{array}$ & $\begin{array}{l}\text { Revisar os benefícios do exercício aeróbico } \\
\text { sobre o rejuvenescimento cardiovascular. }\end{array}$ \\
\hline $\begin{array}{l}\text { Leukocyte telomere length in } \\
\text { healthy caucasian and } \\
\text { african-american adolescents: } \\
\text { Relationships with Race, Sex, } \\
\text { Adiposity, Adipokines, and } \\
\text { Physical Activity. }\end{array}$ & Zhu, H., et al. & J. Pediatr., 2011. & $\begin{array}{l}\text { Examinar as relações entre raça, sexo, } \\
\text { adiposidade e atividade física como a } \\
\text { estrutura do telômero. }\end{array}$ \\
\hline $\begin{array}{l}\text { Exercise in the Elderly: } \\
\text { Research and Clinical } \\
\text { Practice. }\end{array}$ & Frankel, J., et al. & $\begin{array}{l}\text { Clin. Geriatr. Med., } \\
2006 .\end{array}$ & $\begin{array}{l}\text { Revisar os efeitos primários do exercício em } \\
\text { pacientes idosos e suas prescrições } \\
\text { específicas. }\end{array}$ \\
\hline
\end{tabular}

Fonte: Dados do estudo

Vale ressaltar, todavia, que o procedimento de análise ocorreu sobre cada obra em sua totalidade tendo em vista às indicações de Foucault (2008) e Fischer (2001) sobre a necessidade de observarmos nos discursos a presença de: 1) um referente (ou seja, a referência a algo que identificamos nos textos); 2) um sujeito (identificando aqueles que falam por meio dos artigos); 3) um campo associado (quer dizer, os enunciados ou discursos que se articulam nos artigos); 4) uma materialidade específica (isto é, a presença de elementos que materializam o discurso (as tecnologias de rejuvenescimento). 
Tabela 2. Estudos selecionados a partir da pesquisa no portal de Periódicos CAPES.

\begin{tabular}{|c|c|c|c|}
\hline TITULO & AUTOR & REVISTA & OBJETIVOS \\
\hline Growth hormone in aging males. & Sattler, F. & $\begin{array}{l}\text { Best Practice } 8 \\
\text { Research Clinical } \\
\text { Endocrinology } 8 \\
\text { Metabollsm, 2013. }\end{array}$ & $\begin{array}{l}\text { Revisar na IIteratura o potenclal para } \\
\text { aumento do } \mathrm{GH} \text { em homens Idoso. }\end{array}$ \\
\hline $\begin{array}{l}\text { Older women and their } \\
\text { representations of old age: a } \\
\text { qualitative analysis. }\end{array}$ & $\begin{array}{l}\text { Quenlart, A.; } \\
\text { Charpentler, E. }\end{array}$ & $\begin{array}{l}\text { Ageling \& Soclety. } \\
\qquad 2 \mathrm{D} 12 \text {. }\end{array}$ & $\begin{array}{l}\text { Anallsar as representacbes sobre ser } \\
\text { Idoso e envelnecimento em tres } \\
\text { geracolos de ldosas com diferentes } \\
\text { historias de vida. }\end{array}$ \\
\hline $\begin{array}{l}\text { Growth hormone responses to } \\
\text { acute reslstance exerclse with } \\
\text { vascular restriction lin young and } \\
\text { old men. }\end{array}$ & $\begin{array}{c}\text { Maninl, } T_{\text {.. }} \text { et } \\
\text { al. }\end{array}$ & $\begin{array}{l}\text { Growth Hormone } \& \\
\text { IGF Research, } \\
2 \mathrm{D} 12 .\end{array}$ & $\begin{array}{l}\text { Comparar as repostas do horm onlo } \\
\text { do crescimento (GH) frente reallzaçăo } \\
\text { de diferentes protocolos de exercicio } \\
\text { resistido em Individuos jovens e } \\
\text { ldosos. }\end{array}$ \\
\hline $\begin{array}{l}\text { Moderate exerclse training } \\
\text { modulates cytokine profile and } \\
\text { sleep in elderly people. }\end{array}$ & $\begin{array}{c}\text { Santos, R., et } \\
\text { al. }\end{array}$ & Cytokine, 2012 . & $\begin{array}{l}\text { Medir os efeitos do exercicio fislco } \\
\text { modierado no sono em pessoas } \\
\text { Idosas relacionando com seu pernl } \\
\text { Inflamatorio. }\end{array}$ \\
\hline $\begin{array}{l}\text { Pharmacologic Approaches to the } \\
\text { Agling Athlete. }\end{array}$ & $\begin{array}{l}\text { Tokish, J.: } \\
\text { DeRosa, D. }\end{array}$ & $\begin{array}{l}\text { Sports Health: A } \\
\text { Muitidiscipilinary } \\
\text { Approach, 2013. }\end{array}$ & $\begin{array}{l}\text { Revisar as bases clentincas de } \\
\text { produtos antlaging divulgados no } \\
\text { mercado. }\end{array}$ \\
\hline $\begin{array}{l}\text { Obesity and Mobility in Advancing } \\
\text { Age: Mechanlsms and } \\
\text { Interventions to preserve } \\
\text { Independent Mobility. }\end{array}$ & $\begin{array}{l}\text { Heather, } v_{-} \\
\text {Mathews, A. }\end{array}$ & $\begin{array}{l}\text { Current Obesity } \\
\text { Report, } 2013 \text {. }\end{array}$ & $\begin{array}{l}\text { Anallsar a Incapacidade funclonal em } \\
\text { Indlviduos idosos obesos. }\end{array}$ \\
\hline $\begin{array}{l}\text { Errect of tal chl on } \\
\text { musculoskeletal health-related } \\
\text { ntness and self-reported physical } \\
\text { health changes in low income, } \\
\text { multiple ethniclty mid to } \\
\text { older adults. }\end{array}$ & $\begin{array}{c}\text { Manson, J., et } \\
\text { al. }\end{array}$ & $\begin{array}{l}\text { BMC Gerlatrics, } \\
2013 .\end{array}$ & $\begin{array}{l}\text { Anallsar mudanças na aptidăo } \\
\text { musculoesqueletica relacionada a } \\
\text { saude e na saude flslca apos um } \\
\text { programa de TC numa populaçăo } \\
\text { idosa de balxa renda e de etnla } \\
\text { multipla. }\end{array}$ \\
\hline $\begin{array}{l}\text { Successful Images of Successtul } \\
\text { Ageing? Representations of } \\
\text { Vigorous Elderly People In a } \\
\text { Swedish Educational Television } \\
\text { Programme. }\end{array}$ & Walander, $\mathrm{K}$. & $\begin{array}{l}\text { Nordicom Review . } \\
2 \mathrm{D} 13 \text {. }\end{array}$ & $\begin{array}{l}\text { Demonstrar como as representaçठ́des } \\
\text { de idosos vigorosos săo construldas } \\
\text { no programa VeteranTV. pela } \\
\text { televisăo educativa sueca. }\end{array}$ \\
\hline
\end{tabular}

Fonte: Dados do estudo

Esses quatro elementos permitiram identificar enunciados os quais, segundo Foucault (2008), funcionam como funções de existência. Revelar enunciados, portanto, significa perceber como certos discursos promovem modificações da realidade ou instaurações de novos processos de problematização e, consequentemente, inauguração de novos sistemas de verdade.

Após a identificação dos enunciados realizamos uma nova leitura dos textos. Isso permitiu identificar articulações de pensamento as quais revelaram certos aspectos relacionados à genealogia do objeto investigado no que tange a duas questões principais: 1) a maneira como o rejuvenescimento tecnológico do corpo se formou e continua a existir como saber científico; 2) os desdobramentos deste saber sobre a Educação Física.

Nos próximos momentos procuramos discutir as evidências coletadas. Depois, realizamos uma análise das técnicas de rejuvenescimento citadas na literatura científica, indicando seu processo de racionalização, isto é, como os cientistas pensam o rejuvenescimento tecnológico do corpo. No último momento, discutiremos o lugar da Educação Física nesse contexto, apontando alguns desdobramentos na maneira de entender o exercício físico. 


\section{Rejuvenescimento como problema científico: cartografando evidências}

Os estudos que constituíram o corpus da presente investigação possuem informações importantes sobre o tema rejuvenescimento. Para facilitar a compreensão, procedemos em duas etapas. Na primeira, realizamos uma apresentação dos estudos demonstrando aspectos relativos aos sujeitos. $\mathrm{O}$ segundo momento consistiu na análise do referente.

Do ponto de vista dos sujeitos, tratamos de identificar os autores que produziram reflexões sobre o rejuvenescimento tecnológico do corpo para acessar de onde partem as contribuições teóricas sobre o tema no recorte de tempo aqui selecionado. Uma incursão desse tipo serviu para caracterizar quais são os discursos hegemônicos que produzem enunciados verdadeiros dentro da ciência, mesmo porque a própria ciência é constituída por relações de disputa definidoras de posições mais propícias ao exercício de poder (FOUCAULT, 2005).

Verificamos que dos 13 estudos selecionados, todos apresentaram dados sobre a formação acadêmica dos pesquisadores. Em sua maioria, os estudos foram provenientes da área médica. Com isso percebemos que o discurso médico ocupa um lugar significativo nas reflexões sobre rejuvenescimento.

No intuito de operacionalizar uma incursão mais profunda, realizamos um levantamento sobre os objetivos e sobre o escopo dos periódicos nos quais os artigos foram publicados. Iniciando pelos periódicos acessados, percebemos a presença de estudos realizados nas áreas da endocrinologia, metabolismo e inflamação (SATTLER, 2013; SANTOS et al., 2012; STRANAHAN, A. et al., 2012), na geriatria e no envelhecimento humano com direcionamento sociológico (QUÉNIART; CHARPENTIER, 2012), na área de hipertensão, cardiologia e angiologia (PUCCI, G. et al., 2012; CHARANSONNEY, 2012), na endocrinologia (MANINI, 2012), e no campo da ergonomia (VINCENT; MATHEWS, 2013). Identificamos, ainda, um estudo na área de fertilidade (MORLEY, 2013), um estudo na área pediátrica (ZHU, H. et al., 2011), uma investigação na dermatologia (SOUZA, C. et al., 2013), além de estudos nas ciências sociais (MANSON et al. 2013; WALLANDER, 2013) e uma pesquisa interdisciplinar na área esportiva (TOKISH; DE ROSA, 2013).

Em relação aos objetivos de estudo, verificamos textos marcados por intenções teóricas referentes a modelos que explicam o rejuvenescimento por meio de diferentes tecnologias, ou que procuraram articular o rejuvenescimento a intervenções estéticas, funcionais e fisiológicas.

De forma geral, o levantamento de artigos demonstrou a existência de uma estrutura de saberes cuja especialização denota características daquilo que Foucault (2005) denominou "saberes sujeitados". Em outras palavras, os saberes produzidos sobre o rejuvenescimento formam conjuntos funcionais, formais de conceitos que mascaram certos conteúdos históricos precedentes ao saber científico, dando a falsa impressão de que estes sempre foram científicos.

Seguindo esta lógica, a transformação do rejuvenescimento em objeto da ciência não parece ser um processo dado ou aleatório, mas algo inventado. Uma forma de ratificar o argumento da invenção do rejuvenescimento pode ocorrer tomando como exemplo o caminho traçado por Foucault nas suas reflexões sobre loucura, delinquência e sexualidade. Segundo o autor, o aparecimento de cada um destes elementos como objetos científicos foi posterior ao reconhecimento de que eles deveriam ser controlados socialmente. Esse controle se tornou possível pela ação de instituições confessionais e de exame que estimularam os sujeitos a declarar e registrar diferentes aspectos de sua vida íntima. Após isso, essas mesmas 
instituições passaram a determinar limites de normalidade e anormalidade para padronizar condutas em larga escala (FOUCAULT, 2006).

Tal como os exemplos da loucura, delinquência e sexualidade, podemos considerar que o rejuvenescimento, antes mesmo de pertencer à ciência, se manifestou como consequência de discursos médicos sobre o envelhecimento (VELOZ et. al., 1999). Por outro lado, tão inegável quanto o seu caráter científico é o fato de que as sociedades contemporâneas consideram a juventude como referência de felicidade e a velhice como um problema. É o que o estudo de Wallander (2013) demonstrou ao discutir o papel decisivo da mídia na produção de agenciamentos que podem estimular os sujeitos a reproduzir e experimentar diferentes maneiras de viver.

Dessa maneira, podemos identificar que os estudos analisados carregam na sua interioridade elementos reveladores de uma cientificização do rejuvenescimento que opera a partir de dois movimentos: valorização funcional-estética da juventude, e transformação do envelhecimento biológico como um problema da ciência, ou, em termos foucaultianos, problematização do envelhecimento.

No próximo momento procuramos abordar as técnicas de rejuvenescimento encontradas no corpus da pesquisa tentando destacar aspectos de sua racionalização.

\section{Técnicas do rejuvenescer: polissemias de uma racionalização em processo}

Neste momento, nos debruçamos sobre as técnicas de rejuvenescimento descritas na literatura científica analisada. Apesar da especificidade de nosso corpus de estudo, pudemos perceber a existência de várias técnicas as quais tem fundamento em diferentes hipóteses de pesquisa. Por essa razão, a reflexão seguinte é uma discussão sobre a polissemia, não de discursos, mas de tecnologias.

Começamos o estudo sobre a polissemia tecnológica apontando o primeiro grupo de técnicas de rejuvenescimento o qual remete a dois estudos de caráter farmacológico. À primeira vista, eles defendem a administração de medicamentos ou hormônios com a finalidade de promover recuperação de massa muscular e da massa óssea, ou recuperação e melhoria da autonomia física.

Tokish e De Rosa (2013) consideram as terapias farmacológicas como uma estratégia anti-aging, e tecem observações sobre diferentes tecnologias como a reposição hormonal, os moduladores seletivos de receptores androgênicos e de estrógeno, e os antioxidantes como o resveratrol, a quercetina, vitaminas $\mathrm{C}$ e $\mathrm{E}$, a glutationa e a melatonina.

Os autores ainda se referem a uma tecnologia anti-aging baseada na ação de hormônios que são os Selective Estrogen Receptor Modulators (SERMs) - Moduladores Específicos de Receptores de Estrogênio, e os Selective Androgen Receptor Modulators (SARMs) Moduladores Específicos de Receptores Androgênicos.

No grupo dos SERMs destaca-se o Citrato de Clomifeno que é largamente utilizado por mulheres para estimular a ovulação. Porém, recentemente, ele também tem sido utilizado em homens com deficiência hormonal para estimular a produção de testosterona evitando os possíveis efeitos da sua suplementação exógena. O grupo dos SARMs, por sua vez, caracteriza-se por melhorar a sensibilidade dos receptores celulares de testosterona para potencializar seus efeitos biológicos.

Em relação aos tratamentos hormonais, aos SERMs e aos SARMs, interessa ressaltar que as opiniões dos autores referem-se sempre à recuperação do estado hormonal senil 
tomando como referência o estado hormonal normal apresentado por jovens saudáveis. $\mathrm{O}$ termo que melhor representa esse processo é a palavra restaurar. Permeando essa questão, é possível verificar a existência de um processo de normalização que norteia a compreensão de envelhecimento hormonal dos indivíduos senis. Essa normalização, se pensada na perspectiva de Foucault (2004), objetiva classificar e excluir os indivíduos conforme uma referência instituída pelo saber que termina por concretizar uma homogeneização da vida.

O segundo estudo que compreende rejuvenescimento como condição passível de ser farmacologicamente produzida é o de Morley (2013), que consistiu numa revisão sobre os possíveis benefícios do uso de testosterona, $\mathrm{GH}$, melatonina, grelina, dehidroepiandrosterona (DHEA) e pregnenolona no combate ao envelhecimento.

Diferentemente de Tokish e DeRosa, o autor reconhece a potencialidade das terapias hormonais anti-aging, mas apenas num plano teórico. Em sua opinião, nenhuma terapia hormonal pode ser plenamente aceita como estratégia de rejuvenescimento se for utilizada isoladamente (grifo nosso). Ele vai mais além quando passa a defender o exercício físico, a alimentação e a exposição adequada ao sol como maneiras eficazes de obter rejuvenescimento. $\mathrm{O}$ autor termina seu artigo dizendo: "Overall, exercise, adequate sunlight exposure, and adequate protein intake all appear more appropriate "elixirs of youth" than hormone supplementation" (MORLEY, 2013, p. 1810). Dessa forma, Morley defende que o rejuvenescimento pode ser obtido somando-se condutas de cuidado associados ao estilo de vida, ao invés de defender o rejuvenescimento apenas como manipulação hormonal. Eis aí uma ruptura em relação às conclusões de Tokish e DeRosa.

Outro tipo de tecnologia encontrada expressa uma compreensão de rejuvenescimento associada à função imunológica. Mais especificamente, ela se refere ao estudo das condições inflamatórias que podem prejudicar o estado fisiológico dos idosos.

Zhu et al. (2011) conduziram um estudo com indivíduos adolescentes de diferentes etnias no qual se constatou que o rejuvenescimento é um estado adquirido pelo uso de recursos que permitem a preservação do tamanho do telômero, partes do DNA que regulam o envelhecimento celular, independente de raça e sexo.

A mesma relação entre rejuvenescimento e controle dos aspectos imunológicos/ inflamatórios se faz presente no estudo de Santos et al. (2012). Os autores partem da premissa de que a piora na qualidade do sono em idosos está ligada ao aparecimento de inflamações sistêmicas típicas da idade. Sendo assim, dormir mal pode contribuir para o aceleramento do envelhecimento biológico.

O objetivo da investigação foi analisar o uso do exercício físico para melhorar o sono e consequentemente alterar positivamente o quadro imunológico dos sujeitos. Em relação aos resultados, a investigação demonstrou que reduções nos níveis de citocinas (marcadores inflamatórios) como a isoleucina 6 (IL), a isoleucina 10 e o TNF alfa (fator de necrose tumoral alfa), após a intervenção como o exercício físico, melhoraram a qualidade do sono em 22 voluntários idosos, contribuindo para regularização da função imunológica e melhoria da qualidade de vida.

Os problemas da mortalidade e do crescente adoecimento da população idosa associada à perda funcional compõem o cenário apresentado para justificar a investigação. $\mathrm{O}$ reconhecimento velado dos impactos sociais decorrente dessas questões aponta para uma perspectiva biopolítica presente no estudo. As conclusões sobre a necessidade de administrar o sono demonstram a preocupação de manter as condições de saúde necessárias à qualidade de vida, mas também às condições físicas de produzir trabalho. $\mathrm{O}$ instrumento utilizado para 
avaliar a qualidade de vida (SF 36) revela esse fato, visto que a maioria das questões presentes no questionário refere-se à capacidade de realizar trabalho.

O terceiro grupo de tecnologias de rejuvenescimento compreende as estratégias antiaging voltadas à melhoria estética. Souza et al. (2013) realizaram uma revisão sobre as técnicas de rejuvenescimento facial utilizadas pelo campo da motricidade orofacial para melhorar a saúde e a aparência física.

Verifica-se uma ruptura na compreensão de rejuvenescimento, referenciado como correção dos sinais de envelhecimento. No artigo foram citados diferentes recursos terapêuticos como a terapia miofuncional individual, massagens, manobras de alongamento e exercícios orofaciais que possibilitaram resultados como equilíbrio da tensão muscular, suavização das linhas de expressão, rejuvenescimento e suavização da face, conscientização de posturas, olhos mais abertos, redução de bolsas na pálpebra inferior, melhora na pálpebra superior, melhor definição do contorno facial e redução das olheiras.

Nesse caso, não se trata de conceber o rejuvenescimento pelo uso de medicamentos, terapias hormonais, antioxidantes, ou técnicas que permitem a regulação do estado imunológico e genético, mas de utilizar exercícios localizados para o fortalecimento e alongamento dos músculos e consequente melhora estética da face. Como nas situações anteriores, a ruptura não se encontra na finalidade biopolítica que rege a existência das tecnologias, e sim nas diferentes maneiras através das quais o problema do rejuvenescimento pode ser pensado.

\section{Uma transfiguração no saber: o exercício físico como "fonte da juventude"}

Nesse momento, serão destacados os artigos que citaram o exercício físico como método de rejuvenescimento, evidenciando o próprio como um mantenedor ou recuperador das capacidades funcionais.

No estudo Older women and their representations of old age: a qualitative analysis (QUÉNIART; CHARPENTIER, 2012), pode-se observar que para combater o envelhecimento essencialmente visto como degeneração, há uma indicação sobre o controle do estilo de vida por meio de dieta e exercício físico. As mulheres mais velhas que foram entrevistadas transcendem essa definição. Suas representações de "envelhecer bem" e do envelhecimento expressam valores positivos de autonomia, independência, consistência, integridade, manutenção da saúde física e intelectual.

$\mathrm{Na}$ concepção geral dos entrevistados, a expressão "ser uma mulher mais velha" é sinônimo de abrandar, a inatividade, o tédio e o isolamento. Por outro lado, envelhecer evoca doenças, a dependência de medicamentos e a institucionalização, baseando- se que na velhice há uma perda da capacidade física e cognitiva, fragilidade e dependência.

Charansonney (2012) em seu artigo Activité physique et vieillissement: des effets physiologiques opposes, apresenta dois modelos que suportam esses efeitos. O primeiro modelo descreve o caminho para a fragilidade e o segundo explica que o sedentarismo é um estressor que desencadeia estresse - resposta responsável por muitas doenças crônicas. Nesse sentido, o autor defende a hipótese que o treinamento físico aumenta a reserva fisiológica por aqueles que se opõem aos efeitos da idade.

No artigo Growth hormone responses to acute resistance exercise with vascular restriction in young and old men (MANINI, 2012), que teve por objetivo comparar as respostas do hormônio do crescimento $(\mathrm{GH})$ em relação à aplicação de exercício físico 
resistido de baixa intensidade com restrição vascular comparado a exercício resistido de alta intensidade em jovens e idosos saudáveis, observou-se que o exercício físico em alta intensidade aumenta a expressão de GH tanto em jovens quanto em idosos. O exercício físico de baixa intensidade com restrição vascular pode aumentar a expressão do GH em ambos os grupos. No grupo de idosos, o treino com cargas altas apresentou maior liberação aguda de GH.

Verificou-se que o rejuvenescimento pode estar atrelado a uma condição hormonal, atrelada à prática de exercício físico. A este respeito, a administração de GH exógena não promove a acumulação de massa muscular em adultos, mas reduz a gordura visceral e subcutânea em populações deficientes e promove a síntese de colágeno no tecido músculotendinoso. No entanto, os efeitos colaterais associados à administração de GH e sua limitada eficácia na promoção da função muscular em indivíduos mais velhos exclui a sua recomendação como uma terapia antienvelhecimento.

No artigo Moderate exercise training modulates cytokine profile and sleep in elderly people (SANTOS et al., 2012), constatou-se que o treinamento físico moderado pode reduzir as concentrações plasmáticas de citocinas pró-inflamatórias e aumentar as de citocinas antiinflamatórias, consequentemente, melhorando a qualidade do sono.

Este estudo mostra que o treinamento físico moderado pode melhorar aspectos do sono adverso em indivíduos mais velhos, como a latência do sono REM, o tempo de vigília e eficiência do sono. Além disso, esses melhoramentos podem ser parcialmente modulados por modificações no perfil de citocinas e por uma redução da inflamação.

No artigo Effect of tai chi on musculoskeletal health-related fitness and self-reported physical health changes in low income, multiple ethnicity mid to older adults (MANSON et al., 2013), que teve como meta analisar mudanças na aptidão musculoesquelética relacionada à saúde e na saúde física auto relatada após um programa de Tai chi chuan realizado em 16 semanas numa população idosa de baixa renda e de etnia múltipla, não houve nenhuma mudança significativa na massa corporal e dores no corpo.

No entanto, houve melhorias significativas observadas em medidas de saúde musculoesquelética, na força manual (grip), flexão de braço em 30 segundos, sentar e levantar em 30 segundos, no teste de sentar e alcançar, assim como no funcionamento físico, saúde em geral, e na medida de saúde física agregada ao SF 36.

As funções corporais básicas, como força, resistência, equilíbrio e flexibilidade em membros superiores e nos membros inferiores, são importantes para manter independência física na idade mais avançada. Tomando o rejuvenescimento como recuperação e manutenção da independência funcional, essas evidências continuam a apoiar os efeitos benéficos da aptidão física musculoesquelética na prevenção de doenças crônicas em combinação com melhorias no desempenho das atividades da vida diária.

No artigo Scientific overview of hormone treatment used for rejuvenation (MORLEY, 2013), o exercício é apontado como especialmente importante em idosos frágeis. Exercício em pessoas frágeis tem sido utilizado para aumentar o desempenho funcional, aumentar a velocidade de caminhada, a cadeira de carrinho, a subir escadas, o equilíbrio, diminuir a depressão, o medo de cair, a institucionalização, a mortalidade e melhorar a cognição.

Existem também evidências, embora menos fortes, de que a adição de um suplemento de aminoácidos essenciais enriquecida com leucina para o exercício de resistência melhora a função muscular. Sabe-se que o exercício associado à dieta diminui a perda de massa muscular e óssea. A combinação entre exercício, adequada exposição à luz solar, e consumo 
de proteína dietética parece ter um efeito tão positivo para o rejuvenescimento quanto qualquer hormônio a ser utilizado com essa finalidade.

No artigo Aerobic physical exercise and arterial de-stiffening: a recipe for vascular rejuvenation? (PUCCI, G. et al., 2012), que buscou revisar os benefícios do exercício aeróbico sobre o rejuvenescimento cardiovascular, foi constatado que o exercício ajuda a rejuvenescer a função cardíaca reduzindo o enrijecimento das paredes vasculares.

No estudo publicado por ZHU, H. et al. (2011), que teve como objetivo examinar o comprimento de telômeros em relações à raça, sexo, obesidade, adipocinas e atividade física em adolescentes, os resultados sublinham a importância do exercício físico regular para o envelhecimento saudável para pessoas de todas as idades, incluindo adolescentes.

\title{
Considerações finais
}

Dado que a biopolítica não somente desempenha efeitos no nível das políticas de estado, mas também, determinam relações microfísicas que influenciam os sujeitos a investirem sobre eles, potencializando assim, suas capacidades produtivas, acreditamos que em relação ao rejuvenescimento, a ciência tem alimentado a visão do corpo como máquina e explorado a manipulação deste, acreditando na possibilidade de construção de um corpo que resiste ao envelhecimento.

Levando-se em conta o que foi observado, a presente investigação concluiu que o rejuvenescimento tornou-se um objeto de estudo científico com o objetivo de recuperar, melhorar, retardar os sinais de declínio, levantando assim, diversificadas formas para controlar o corpo desses sinais.

\section{BIOPOLITICS E REJUVENATION: A SYSTEMATIC REVIEW ON SCIENTIFIC TALKS IN PHYSICAL EDUCATION}

\begin{abstract}
It was proved that physical education has been linked to the biopolitical speeches of rejuvenating power effects in the measure as it offers us the possibility of building a body sturdy aging. The scientific discourses of Physical Education seem to support the establishment of links between knowledge and practice of power on rejuvenation. Thus, the following systematic review aims to analyze the process of formation of physical education as a discourse and practice of power over the rejuvenation, identifying the narratives about body rejuvenation in scientific journals, and checking how these narratives are linked to knowledge of physical education.
\end{abstract}

Keywords: Biopolitics. Rejuvenation. Physical Education.

\section{BIOPOLÍTICA Y REJUVENECIMIENTO: UNA REVISIÓN SISTEMÁTICA SOBRE LOS DISCURSOS CIENTÍFICOS EN LA EDUCACIÓN FÍSICA}

\section{Resumen}

Se descubrió recientemente que la Educación Física se ha relacionado con los discursos biopolíticos de rejuvenecimiento desempeñando efectos de poder en la medida que nos oferta la posibilidad de construir un cuerpo resistente al envejecimiento. Los discursos científicos de la Educación Física parecen corroborar para el establecimiento de vínculos entre los conocimientos y prácticas de poder 
sobre el rejuvenecimiento. Por lo tanto, la siguiente revisión sistemática tiene como objetivo analizar el proceso de formación de la Educación Física como discurso y práctica de poder sobre el rejuvenecimiento, la identificación de las narrativas del rejuvenecimiento del cuerpo en las revistas científicas, y comprobar cómo estos relatos están relacionados con el conocimiento de la Educación Física.

Palabras clave: Biopolítica. Rejuvenecimiento. Educación Física.

\section{Referências}

CHARANSONNEY, O. Activité physique et vieillissement: des effets physiologiques opposés. Annales de Cardiologie et d'Angéiologie, v. 61, 2012. p. 365-369.

COSTA, E.; VENÂNCIO, S. Atividade física e saúde: discursos que controlam o corpo. Pensar a Prática, v. 7, n.1, mar. 2004. p. 59-74.

CRAIG, B. W.; BROWN, R.; EVERHART, J. Effects of progressive resistance training on growth hormone and testosterone levels in young and elderly subjects, Mech. Ageing. Dev., v. ;49, n. 2, 1989. p. 159-69.

CUSSONS, A.; BHAGAT C.; FLETCHER S.; WALSH J. Brown-Séquard revisited: a lesson from history on the placebo effect of androgen treatment. Med J Aust., v. 2-16, n. 177, p. 1112:678-9, dez., 2002.

FISCHER, R. Foucault e a análise do discurso em educação. Cadernos de Pesquisa, n. 114, p. 197-223, nov., 2001.

FOUCAULT, M. Vigiar e Punir. Petrópolis: Vozes, 2004.

A arqueologia do saber. Rio de Janeiro: Forense Universitária, 2008.

A história da sexualidade I: a vontade de saber. São Paulo: Graal, 2006.

HEIDEGGER, M. A questão da técnica. scientiæ zudia, São Paulo, v. 5, n. 3, 2007. p. 37598.

LIBERALI, R. Metodologia científica prática: um saber-fazer competente da saúde à educação. 2. ed. rev. e ampl. Florianópolis: Postmix, 2011.

MANINI, T.; YARROW, J.; BUFORD, T.; CLARK, B.; CONOVER, C.; BORST, S. Growth hormone responses to acute resistance exercise with vascular restriction in young and old men. Growth Hormone \& IGF Research, v. 22, 2012. p. 167-172.

MANSON, J.; ROTONDI, M.; JAMNIK, V. ; ARDERN, C.; TAMIM, H. Effect of tai chi on musculoskeletal health-related fitness and self-reported physical health changes in low income, multiple ethnicity mid to older adults. BMC Geriatrics, n. 28, v.13, 2013. p.114. 
MARIEL, A. La construcción de identidades corporales alternativas en los límites de un proyecto educativo hegemônico. Movimento, Porto Alegre, v. 17, n. 04, p. 83-100, out./dez. 2011.

MORLEY, J. Scientific overview of hormone treatment used for rejuvenation. Fertility and Sterility, v. 99 , n. 7 p. 1807-13, jun., 2013.

NAKAJIMA, T.; YASUDA, T.; SATO, Y.; MORITA, T.; YAMASOBA, T. Effects of Exercise and Anti-Aging. Anti-Aging Medicine, v. 8, n. 7, p. 92-102, 2011.

PUCCI, G.; RECH, C.; FERMINO, R.; REIS. R. Associação entre atividade física e qualidade de vida em adultos. Rev Saúde Pública, v. 46, n. 1, 2012. p. 166-79.

QUÉNIART, A.; MICHÈLE CHARPENTIER, M. Older women and their representations of old age: a qualitative analysis. Ageing and Society, v. 32, 2012. p. 983-1007.

SANTOS, R.; VIANA, V.; BOSCOLO, R.; MARQUES, V.; SANTANA,M.; LIRA, F.; TUFIK, S.; MELLO, M. Moderate exercise training modulates cytokine profile and sleep in elderly people. Cytokine, v. 60, 2012. p.731-735.

SATTLER, F. Growth hormone in the aging male. Best Practice \& Research Clinical Endocrinology \& Metabolism, v. 27, 2013. p. 541-555.

SOUZA. C.; GUERRA, J.; BARBOSA, M.; PORTO, C. Rejuvenescimento facial por intervenção miofuncional estética. Revisão integrativa. Med Cutan Iber Lat Am, v. 41, n. 4, 2013. p. $165-171$.

STRANAHAN, A.; MARTIN, B.; CHADWICK, W.; PARK, S.; WANG, L.; BECKER, K.; WOOD, W.; ZHANG, Y.; STUART, M. Metabolic context regulates distinct hypothalamic transcriptional responses to antiaging interventions. International Journal of Endocrinology, set./ out., 2012.

TAKAYAMA, K.; AYABE, M.; HAMADA,T.; KIMURA, T.; MORITANI,T.; TANAKA, H.; ISHII, K. The Effects of 12-week Home-Based Bench Stepping Exercise on Aerobic Capacity and Cardiac Autonomic Nervous Activities in Older Adults. AntiAging Medicine, v. 9, n. 7, p. 196-200, 2012.

TOKISH, J.; DE ROSA, D. Pharmacologic Approaches to the Aging Athlete. Sports Health: A Multidisciplinary Approach, v. 20, n. 10, 2013.

VELOZ, M. C. T.; NASCIMENTO-SCHULZE, C. M.; CAMARGO, B. V. Representações sociais do envelhecimento. Psicol. Reflex. Crit., v.12, 1999. p.479-501.

VINCENT, H.; MATHEWS, A. Obesity and Mobility in Advancing Age: Mechanisms and Interventions to Preserve Independent Mobility. Curr Obes Rep, v. 2, 2013. p.275-283. 
WALLANDER, K. Successful Images of Successful Ageing? Representations of Vigorous Elderly People in a Swedish Educational Television Programme. Nordicom Review, v. 34, n. 1, 2013. p. 91-103.

WEBER, M. A ética protestante e o espírito do capitalismo. São Paulo: Martin Claret, 2004.

WENETZ, I.; STIGGER, M.; MEYER, D. As (des)construções de gênero e sexualidade no recreio escolar. Revista Brasileira de Educação Física Esporte, São Paulo, v. 27, n. 1, p. 117-128, jan./mar. 2013.

ZHU, H.; MATTHEW, M.; HARST, P. Healthy aging and disease: role for telomere biology? Clin Sci, London, v. 1, n. 120, p. 427-440, maio 2011

Recebido em: 27/07/2015

Revisado em: 01/09/2015

Aprovado em: 15/09/2015

Endereço para correspondência:

ritaspidanca@ig.com.br

Rita de Cássia Paulino Silva

Universidade Federal da Paraíba, Departamento de Educação Física.

Centro de Estudos da Saúde

Cidade Universitária

58051-900 - João Pessoa, PB - Brasil 